\title{
Empatía y habilidades comunicativas en profesionales de la salud que trabajan con enfermedades crónicas pediátricas
}

\section{Empathy and communication skills in health professionals who work with pediatric chronic diseases}

\author{
Ivana Raba ${ }^{1}$, Rocío A. Hauché ${ }^{2}$ \\ Universidad Abierta Interamericana \\ Lucas G. Gago Galvagno ${ }^{3}$ \\ Universidad de Buenos Aires
}

Recibido: $12-04-21$

Aceptado: $07-05-21$

Publicado: $18-06-21$

\section{Resumen}

Las enfermedades crónicas pediátricas tienen una alta tasa de prevalencia en Argentina, sin embargo, poco se ha estudiado acerca de las competencias profesionales necesarias para la intervención en estos casos, que resultan ser importantes para alcanzar objetivos terapéuticos. El objetivo de este estudio fue indagar la relación entre la empatía y las habilidades comunicativas en profesionales de la salud que trabajan con pacientes pediátricos con enfermedades crónicas. El muestreo fue por conveniencia y compuesta por 146 profesionales de la salud de género femenino $(M=37,22 ; D E=9,83)$. Las variables son evaluadas de manera online a través del Índice de Reactividad Interpersonal, la Escala de Habilidades Comunicativas en Profesionales de la Salud y un Cuestionario Sociodemográfico ad-hoc. Los resultados alcanzados señalan una correlación parcial entre las variables de estudio ya que, si bien los constructos totales de empatía y habilidades comunicativas no han correlacionado significativamente, sí es posible observar una asociación significativa entre las siguientes subdimensiones: por un lado, toma de perspectiva (empatía) y respeto (habilidades comunicativas) ( $\mathrm{r}=$ .193) y, por el otro, malestar personal y habilidades sociales $(r=.274)$, componentes de la empatía y de las habilidades comunicativas respectivamente . Como conclusión, se resalta el valor de los vínculos construidos con los pacientes pediátricos que, sostenidos sobre la base de un desempeño profesional asertivo empático y comunicativo, podría convertirse en un potencial recurso terapéutico.

1 Licenciada en Psicología de la Universidad Abierta Interamericana (UAI) Buenos Aires, Argentina. Cursando actualmente la especialización en Psicoterapias Cognitivas Contemporáneas (Fundación Foro). Autor para correspondencia: ivana.raba@yahoo.com.ar ORCID: https://orcid.org/0000-0001-8316-5430

2 Licenciada en Psicología de la Universidad Abierta Interamericana (UAI). Buenos Aires, Argentina. E-mail: hauche.rocio@gmail.com ORCID: https://orcid.org/0000-0001-8821-115X

3 Profesor y Licenciado en Psicología en Universidad de Buenos Aires, Argentina (UBA). Doctor en Psicología en Universidad Católica Argentina (UCA).

E-mail: lucas.gagogalvagno@hotmail.com ORCID: https://orcid.org/0000-0001-5993-3866

(C) Los autores. Este artículo es publicado por la Revista de Investigación en Psicología de la Facultad de Psicología, Universidad Nacional Mayor de San Marcos. Este es un artículo de acceso abierto, distribuido bajo los términos de la licencia Creative Commons Atribución 4.0 Internacional (CC BY 4.0) [https://creativecommons.org/licenses/by/4.0/deed.es] que permite el uso, distribución y reproducción en cualquier medio, siempre que la obra original sea debidamente citada de su fuente original. 
Palabras clave: empatía; habilidades comunicativas; enfermedades crónicas pediátricas; profesionales de la salud.

\begin{abstract}
Pediatric chronic diseases have a high prevalence rate at the national level, however, little has been studied about the professional competencies necessary for intervention in these cases, which turn out to be important to achieve therapeutic goals. The objective of this study was to investigate the relationship between empathy and communication skills in health professionals who work with pediatric patients with chronic diseases. The sample is for convenience made up of 146 female health professionals $(M=37.22 ; S D=9.83)$. The variables are evaluated online through the Personal Reactivity Index, the Scale of Communication Skills in Health Professionals and an ad-hoc Sociodemographic Questionnaire. The results achieved indicate a partial correlation between the study variables since, although the total constructs of empathy and communication skills have not correlated significantly, it is possible to observe a significant association between the following subdimensions: on the one hand, perspective taking (empathy) and respect (communication skills) $(\mathrm{r}=.193)$ and, on the other hand, personal discomfort and social skills $(\mathrm{r}=.274)$, components of empathy and communication skills respectively. In conclusion, the value of the links built with pediatric patients is highlighted, which, sustained on the basis of an assertive, empathic and communicative professional performance, could become a potential therapeutic resource.
\end{abstract}

Keywords: empathy; communication skills; pediatric chronic diseases; health professionals. 


\section{INTRODUCCIÓN}

Las enfermedades crónicas (en adelante, EC) son enfermedades de larga duración (i.e., más de tres meses) y lenta progresión que alteran las actividades habituales de un sujeto produciendo insuficiencia, incapacidad o minusvalía. Si bien se trata de una condición que puede presentarse en cualquier momento de la vida, cuando afecta a niños se les llama Enfermedades Crónicas Pediátricas (en adelante, ECP) e incluye enfermedades físicas como así también trastornos psicológicos (Quesada Conde et al., 2014; Lejarraga, 2006). De acuerdo a la Organización Mundial de la Salud (2018), las enfermedades crónicas son las responsables de 41 millones de muertes por año en el mundo, lo que corresponde a un $71 \%$ de las defunciones anuales y, aunque solo un $4 \%$ se dan en menores de 30 años de edad, no debe desestimarse la importancia que estas enfermedades tienen en la población infantil.

Considerando las enfermedades de interés en este estudio corresponde señalar que, según el Hospital de Pediatría Garrahan (2010), existen en Argentina entre 500.000 y 2.000 .000 de pacientes pediátricos con enfermedades crónicas. El asma constituye la enfermedad crónica de mayor frecuencia existiendo una prevalencia del 10\% a nivel nacional. Por su parte, 500.000 niños alrededor del mundo tienen diabetes y, en Argentina, entre 5 y 10 niños de cada 100.000 reciben dicho diagnóstico anualmente. Por otro lado, 7.000 menores nacen en dicho país con cardiopatías congénitas, convirtiéndola en una de las anomalías más frecuentes de observar, además de ser la principal causa de muerte en menores de un año. Según la Liga Argentina contra la Epilepsia (2020), ésta tiene una incidencia menor siendo tan solo del $1 \%$ en la población pediátrica argentina. Por último, el Registro Oncopediátrico Hospitalario Argentino (2018) indica que existe un promedio de 1.300 casos oncológicos pediátricos anuales.

En lo que respecta a las enfermedades crónicas psicológicas, no hay en Argentina estadísticas claras ni actualizadas, aunque existe consenso entre los especialistas al considerar que las cifras son similares a las de Estados Unidos, en donde los Trastornos Generalizados del Desarrollo han aumentado un $700 \%$ en los últimos veinte años. Allí, 1 de cada 68 niños recibe el diagnóstico de Autismo en la actualidad (Universidad Favaloro, 2016). La Asociación Argentina de Pediatría (2018) señaló que, aproximadamente, un 1\% de la población infantil presenta TEA. Por su parte, la Universidad Favaloro (2017) indica que alrededor de un 5\% de los menores de 18 años tiene TDAH, equivalente a dos casos por cada 20 niños.

Por otro lado, Rogers (1951) fue uno de los primeros en resaltar la importancia de la empatía en el proceso terapéutico. Señala que se trata de una actitud básica del terapeuta por medio del cual éste refleja una comprensión absoluta de la vivencia del paciente. Actualmente, las terapias basadas en la evidencia apoyan estas ideas al postular que las terapias funcionan por lo que tienen en común más que por sus características específicas, siendo el encuentro interpersonal con el 
paciente el principal factor de cambio (Lambert, 2013; Echeburúa et al., 2010). Yalom (2002) coincide con estas ideas al señalar que lo que más recuerdan los pacientes de su encuentro con los terapeutas es la relación y no las intervenciones realizadas, que en general son más memorables para quien las construyó. La empatía se presenta como habilidad importante para alimentar el vínculo terapéutico con los pacientes y su inicio está determinado por una actitud del profesional dirigido a escuchar y comprender al otro sin juicios previos y con una genuina intención de ayudarlo mientras ha de sostener la neutralidad (Borrell Carrió, 2011). Garantizar un tratamiento adecuado y humano requiere que la empatía se traduzca en conductas observables por medio de comportamientos que le hacen notar al paciente que su comprensión es genuina. Por ende, la comunicación se presenta como una gran aliada de la empatía en la búsqueda del bienestar del cliente (Bermejo, 2012; Mercer y Reynolds, 2002).

Investigaciones previas han demostrado que existe entre la empatía y las habilidades comunicativas una relación positiva y significativa en una muestra de internos en medicina de Perú. Pese a la correlación, el desempeño en habilidades comunicativas es menor al esperado siendo que en el $84,62 \%$ de la muestra el nivel es regular, observando en las subdimensiones empatía y habilidad social mayores debilidades. Por su parte, el grado de empatía puede describirse como medio en el 89.74\% de los casos (Monteza Olivera, 2018).

Las habilidades comunicativas funcionan como factor protector ante el Síndrome de Burnout en profesionales sanitarios, tras observar una correlación negativa con las dimensiones agotamiento emocional y despersonalización y una relación positiva con la dimensión realización personal en el trabajo. De igual manera, la edad y los años de experiencia alcanzaron una correlación negativa con las dimensiones del constructo en el ámbito de atención primaria (Leal Costa et al., 2015). Se ha observado que los entrenamientos en habilidades comunicativas en estudiantes de ciencias de la salud son altamente eficaces para promover un aumento en los niveles de dichas competencias, afectando positivamente no solo la autopercepción de cada profesional en formación sino también el ejercicio de la profesión, siendo médicos y enfermeros quienes puntúan más alto en habilidad social a comparación de auxiliares de enfermería (Leal Costa et al., 2015; Tiuraniemi et al., 2011). Pese a esto, investigaciones previas estudiaron el proceso comunicativo entre pacientes asmáticos infantiles y el personal sanitario y sus resultados arrojan que el $90 \%$ de las interacciones se dan entre el médico y los padres y sólo en el $10 \%$ de las situaciones restantes se ha decidido incluir al niño. Asimismo, un $62 \%$ de las consultas fueron interrumpidas por factores externos y un gran porcentaje de la información fue recogida en la admisión, restando dedicación y tiempo de calidad a la atención personalizada (De la Iglesia et al., 2012). Según Wyatt et al. (2015), en pediatría, sólo un 7\% de las intervenciones se realizan con niños, un $6.11 \%$ con niños y padres por igual y un predominante $63 \%$ únicamente con 
padres. Frente a estos resultados, investigaciones en el área señalan que un 70\% de niños oncológicos, sus padres y pacientes pediátricos que han sobrevivido al cáncer resaltan el valor de que la información sea explicada conjuntamente a niños y adultos. A su vez, hicieron hincapié en la inclusión de los menores en la toma de decisiones y en la necesidad de una postura empática por parte del profesional (Zwaanswijk et al., 2011).

Por otra parte, investigaciones sobre empatía señalan que terapeutas y médicos ya recibidos presentan niveles más bajos de angustia personal que estudiantes de psicología. A su vez, el grupo de terapeutas ha evidenciado un mayor nivel de empatía cognitiva que estudiantes de medicina y mejores resultados en teoría de la mente en comparación al total de estudiantes de medicina y psicología incluidos en la muestra, aunque la exposición clínica (mayor cantidad de años de experiencia y de pacientes atendidos semanalmente) se traduce en una menor puntuación de empatía afectiva producto de un fortalecimiento en las habilidades de regulación emocional (Putrino et al., 2018). Un estudio realizado sobre terapeutas especializados en TEA señala que, quienes trabajan desde el modelo integrativo se desempeñan con mayor compromiso y emocionalidad a diferencia de quienes adhieren a marcos de orientación cognitivo conductual (Casari et al., 2017). Investigaciones realizadas sobre una muestra de estudiantes de carreras sanitarias, afirman que los médicos presentan puntuaciones más altas en la dimensión toma de perspectiva y más bajas en la dimensión compasión del constructo empatía, observando que esta última correlaciona positivamente con la edad. En el caso de los terapeutas, la edad de estudiantes de maestría y psicólogas ya recibidas correlacionó de manera positiva con toma de perspectiva, dimensión que también se relaciona significativamente con la experiencia de profesionales con mayor cantidad de años de trayectoria (Georgi et al., 2015; Mejía de Díaz, 2012).

Teniendo en cuenta estos antecedentes y considerando la escasez de investigaciones sobre la temática y los datos epidemiológicos, los objetivos del presente estudio fueron: describir los niveles de empatía y las habilidades comunicativas en profesionales de la salud que trabajan con ECP, asociar estos constructos entre sí y relacionarlos con las siguientes variables sociodemográficas: edad, categoría profesional, años de experiencia, tipo de terapia, tipo de ECP y atención a niños o a niños y cuidadores. Se sostiene la hipótesis de que la empatía tendrá relación significativa y positiva con las habilidades comunicativas en la población de interés. A su vez, se espera encontrar diferencias según variables sociodemográficas. Los resultados obtenidos permitirán abastecer con valiosa información un área de insuficiente literatura científica, además de beneficiar a profesionales y pacientes en el afán de lograr que la empatía y las habilidades comunicativas sean utilizadas de manera tal que aseguren la protección de la salud y una mejora en la calidad de vida de todos los individuos en juego. 


\section{MÉTODO}

\section{Diseño}

Se empleó un diseño cuantitativo, no experimental (ya que no se manipuló la variable independiente y no hubo control total de las variables contaminadoras), con alcance descriptivo y asociativo, y de corte transversal.

\section{Participantes}

La muestra estuvo compuesta por 146 profesionales de la salud de género femenino que trabajan con enfermedades crónicas pediátricas $(M=37.22 ; D E=9.83)$. En esta investigación se contemplaron participantes que trabajan con los siguientes diagnósticos: Asma (2.7\%), Diabetes (2.1\%), Cardiopatía Congénita, Epilepsia (0.7\%) y Cáncer (5.5\%) como patologías físicas integrantes del grupo de ECP, junto con dos Trastornos del Desarrollo: Trastorno del Espectro Autista [TEA] $(10.3 \%)$ y Trastorno por Déficit de Atención con Hiperactividad [TDAH] $(2.7 \%)$ como ECP psicológicas, habiendo adoptado para su selección un muestreo de carácter no probabilístico por conveniencia. Del total de participantes que componen la muestra, un $90.4 \%$ es de nacionalidad argentina mientras que un $9.6 \%$ corresponde a mujeres extranjeras.

En cuanto a la formación profesional, la mayoría de los participantes eran de Psicología, aplicaban terapia Cognitivo-Conductual, tenían menos de 10 años de experiencia, atendían entre 2 y 4 de las EPC contempladas y se dirigían hacia niños/as y cuidadores por igual. Los datos sociodemográficos específicos de los participantes se resumen en la Tabla 1.

\section{Instrumentos}

Cuestionario sociodemográfico (ad-hoc). Se recabó la edad, formación profesional, tipo de terapia, años de experiencia, tipo de enfermedad crónica infantil con la que trabajan diariamente y atención a niños/as o a niños/as y cuidadores.

Índice de Reactividad Interpersonal [IRI ] (Davis, 1980). Sus propiedades psicométricas fueron validadas en Argentina por Müller et al. (2015). Presentó niveles adecuados de consistencia interna en sus subescalas, validez de constructo y discriminante con respecto a la escala de orientación a la dominancia social (Etchezahar et al., 2014). Se trata de un instrumento autoadministrable compuesto por 28 ítems que se concentra en los componentes cognitivos y afectivos de la empatía. El primero de ellos, es evaluado a través de las siguientes dos dimensiones: Toma de Perspectiva (habilidad para comprender el punto de vista de otra persona) [ $\alpha$ de Cronbach .68] y Fantasía (capacidad imaginativa de ponerse en situaciones ficticias) [ $\alpha$ de Cronbach .72]. Por su parte, la empatía afectiva incluye las dimensiones: Preocupación Empática (compasión, preocupación y 
cariño ante el malestar ajeno) [ $\alpha$ de Cronbach .65] y Angustia Personal (ansiedad y angustia propia ante las experiencias negativas del otro) [ $\alpha$ de Cronbach .73]. El formato de respuesta es de tipo Likert y los valores se extienden de 1 a 5 según el grado de acuerdo o desacuerdo con cada afirmación. A mayor puntaje, mayores serán los niveles de empatía. Los valores de Alpha de las subescalas para esta muestra oscilaron entre .74 y .88 .

Tabla 1

Datos sociodemográficos de la muestra

\begin{tabular}{|c|c|c|c|}
\hline Variable & Etiqueta & $\mathbf{N}$ & Porcentaje \\
\hline \multirow{2}{*}{ Nacionalidad } & Argentina & 132 & 90.4 \\
\hline & Extranjero & 14 & 9.6 \\
\hline \multirow[t]{5}{*}{ Formación Profesional } & Psicóloga & 64 & 43.8 \\
\hline & Médica Pediatra & 15 & 10.3 \\
\hline & Enfermera & 10 & 6.8 \\
\hline & Psiquiatra & 2 & 1.4 \\
\hline & Otro & 55 & 37.7 \\
\hline \multirow[t]{4}{*}{ Tipo de Terapia } & Cognitivo- Conductual & 41 & 64.1 \\
\hline & Psicoanalítica & 11 & 17.2 \\
\hline & Sistémica & 2 & 3.1 \\
\hline & Otras & 7 & 10.9 \\
\hline \multirow[t]{2}{*}{ Años de Experiencia } & Menos de 10 años & 105 & 71.9 \\
\hline & Más de 10 años & 41 & 28.1 \\
\hline \multirow[t]{4}{*}{ ECP que atienden } & Entre 2 y 4 & 81 & 55.5 \\
\hline & Entre 5 y 6 & 13 & 8.9 \\
\hline & Todas & 5 & 3.4 \\
\hline & Otras & 12 & 8.2 \\
\hline \multirow[t]{2}{*}{ Atención a niños o niños y cuidadores } & Sólo niños & 33 & 22.6 \\
\hline & Niños y cuidadores & 113 & 77.4 \\
\hline
\end{tabular}

Nota: ECP: enfermedades crónicas pediátricas.

Escala sobre Habilidades Comunicativas en Profesionales de la Salud [EHC-PS] creada en España (Leal Costa et al., 2016). Se trata de una escala con una adecuada propiedad psicométrica en el análisis de sus ítems. A su vez, se encontró una adecuada validez de constructo medida a través del análisis factorial confirmatorio, una mediana consistencia interna ( $\alpha$ de Cronbach) en sus dimensiones, estabilidad temporal test-retest, y claras evidencias de validez convergente con la Escala de Habilidades Sociales (Gismero, 2010) y de validez discriminante con el Inventario de Burnout de Maslach (Gil-Monte, 2005). Este instrumento tiene como principal objetivo evaluar las habilidades de comunicación de los profesionales de la salud y sus componentes. Éstos conforman las 
cuatro dimensiones que se evalúan en el instrumento: Comunicación Informativa (habilidad para obtener y proporcionar información) [ $\alpha$ de Cronbach .78], Empatía (capacidad de comprender los sentimientos de los pacientes y hacerlo evidente en la relación junto con la correspondiente actitud empática ) [ $\alpha$ de Cronbach .77], Respeto (hace referencia al respeto que muestra el profesional hacia el paciente) [ $\alpha$ de Cronbach .74] y Habilidad Social (destreza para ser asertivo o tener conductas socialmente habilidosas) [ $\alpha$ de Cronbach .65]. Dichas dimensiones conforman un cuestionario autoadministrable de 18 ítems que se responden sobre la base de una Escala Likert que varía desde 1 "Casi Nunca" hasta 6 "Muchísimas Veces" considerando que, a mayor puntaje, mayor será el nivel de Habilidades Comunicativas. Los valores de Alpha de las subescalas para esta muestra oscilaron entre .79 y .92 .

\section{Procedimiento}

La recolección de datos se realizó a través de Google Forms. Los participantes fueron reclutados a través de redes sociales, dejando un medio de contacto en caso de que los sujetos presentaran preguntas o inconvenientes al momento de responder el cuestionario.

La administración de los instrumentos, llevada a cabo en el transcurso de los meses de Septiembre y Octubre del 2020, se realizó de manera individual y cada protocolo contaba con su respectivo consentimiento informado asegurando a los participantes el anonimato de su identidad como así también la preservación y confidencialidad de la información relevada. En cuanto a los aspectos éticos, la investigación fue aprobada por el Comité de Ética de la Universidad Abierta Interamericana y se realizó siguiendo los principios de la Declaración de Helsinki (2000) para investigación médica en seres humanos, incluida la investigación del material humano y de información identificables. Se presentaron los cuestionarios en el mismo orden para todos los participantes, de modo tal que se pudiera realizar un control por equiparación y equilibrar el efecto fatiga y aprendizaje: se comenzó por la encuesta sociodemográfica, luego el Índice de Reactividad Interpersonal y, por último, la Escala de Habilidades Comunicativas en Profesionales de la Salud respectivamente. Los participantes tardaron aproximadamente 15 minutos en completar los cuestionarios.

\section{Análisis de datos}

Se realizó un análisis descriptivo de las principales variables de estudio y, a continuación, se evaluó la normalidad de los datos y sus dimensiones utilizando la prueba Shapiro Wilk y la homogeneidad de varianzas a través de la prueba de Levene. La distribución de las variables fue no normal y se encontró homogeneidad de varianzas, por ende, se emplearon estadísticos no paramétricos. Para valorar la asociación entre variables se utilizó la prueba de correlación Rho de Spearman 
y, para comparaciones múltiples, se utilizó el análisis H de Kruskall-Wallis . En todos los casos se consideró significativo un $p<.05$. Para todo ello, se empleó el programa estadístico SPSS versión 25 de IBM.

\section{RESULTADOS}

\section{Descripción de las Variables}

En la totalidad de los profesionales encuestados, en base a los rangos de las variables medidas, predomina un nivel medio en las dimensiones y constructo total de Empatía. Sin embargo, la variable Toma de Perspectiva puntuó más alto . Por su parte, los puntajes reflejados en la tabla dan cuenta de un nivel alto en cada una de las dimensiones como así también en el constructo total de Habilidades Comunicativas. Los resultados se resumen en la Tabla 2.

Tabla 2

Descripción de los Niveles de Empatía y Habilidades Comunicativas

\begin{tabular}{lccc}
\hline Variable & Media (DE) & $\mathbf{9 5 \%}$ IC & Rango \\
\hline Índice de Reactividad Interpersonal (IRI) & & & \\
\hline Toma de Perspectiva & $23.06(2.91)$ & {$[22.58-23.53]$} & $14-29$ \\
Fantasía & $17.82(3.65)$ & {$[17.22-18.41]$} & $11-28$ \\
Preocupación Empática & $21.77(2.57)$ & {$[21.35-22.19]$} & $14-28$ \\
Malestar Personal & $19.49(3.46)$ & {$[18.92-20.06]$} & $12-31$ \\
Empatía Total & $82.15(7.81)$ & {$[80.87-83.42]$} & $63-105$ \\
\hline Escala sobre Habilidades Comunicativas en Profesionales de la Salud (EHS-PS) & \\
\hline Comunicación Informativa & $28.09(3.49)$ & {$[27.52-28.66]$} & $14-36$ \\
Empatía & $27.39(3.00)$ & {$[26.89-27.88]$} & $17-30$ \\
Respeto & $16.28(1.96)$ & {$[15.96-16.60]$} & $10-18$ \\
Habilidad Social & $15.00(3.40)$ & {$[14.45-15.56]$} & $58-102$ \\
Habilidad Comunicativa Total & & & \\
\hline
\end{tabular}

Nota. IC: Intervalo de Confianza

\section{Asociación entre las Variables de Estudio}

Se observa una relación estadísticamente significativa entre la dimensión Respeto del constructo Habilidades Comunicativas y la dimensión Toma de Perspectiva de la variable Empatía $(r=.193, \mathrm{p}<.05)$, con tamaño del efecto bajo ( $\mathrm{rs} 2=.03)$. A su vez, también se encontró un vínculo significativo entre la dimensión Habilidad Social que compone a las habilidades comunicativas y la dimensión Malestar Personal del constructo Empatía ( $r \mathrm{~s}=.274, \mathrm{p}<.000$ ), con tamaño del efecto bajo ( $\mathrm{rs} 2=.07)$. En ambos casos la correlación es directa, lo cual implica que a medida que una de ellas aumenta, la otra también lo hace. Los resultados se resumen en la Tabla 3. 
Tabla 3

Relación entre Empatía y Habilidades Comunicativas

\begin{tabular}{lccccc}
\hline Variable & $\begin{array}{c}\text { Comunicación } \\
\text { Informativa }\end{array}$ & Empatía & Respeto & Habilidad Social & $\begin{array}{c}\text { Hab. Comunicativa } \\
\text { Total }\end{array}$ \\
\hline Toma de Perspectiva & .107 & .112 & $.193^{*}$ & -.081 & .070 \\
Fantasía & .110 & .029 & .018 & .096 & .097 \\
Preocupación Empática & .119 & .026 & .046 & .025 & .095 \\
Malestar Personal & .05 & -.121 & -.086 & $.274 * *$ & .055 \\
Empatía Total & .140 & -.011 & .020 & .132 & .106 \\
\hline
\end{tabular}

Nota. Hab.: habilidad. Las correlaciones Rho de Spearman fueron reportadas para todas las variables. ${ }^{*} \mathrm{p}<.05$. ${ }^{* * *} \mathrm{p}<.01$.

\section{Análisis de las Diferencias en los Constructos Estudiados en función de la edad, Categoría Profesional, Años de Experiencia y Atención a Niños o Niños y Cuidadores}

Con respecto a la edad, existe una relación estadísticamente significativa y negativa entre la edad y las dimensiones Fantasía ( $\mathrm{rs}=-.172$, $\mathrm{p}<.05$ ) y positiva con Preocupación Empática ( $r s=.201, \mathrm{p}<.05)$ del constructo Empatía. En lo que respecta al constructo Habilidades Comunicativas, se observa una relación significativa y directa únicamente entre la edad y la dimensión Habilidad Social $(\mathrm{rs}=.163, \mathrm{p}<.05)$ siendo que, a mayor edad, mayor destreza social.

En cuanto a la Categoría Profesional, se observan diferencias significativas en la dimensión Malestar Personal (Empatía), siendo mayor en el grupo de pediatras que trabajan con niños con enfermedades crónicas $(\mathrm{H}=8.58, \mathrm{p}<.05)$. A su vez, se hallaron diferencias significativas entre la dimensión Habilidad Social (Habilidades Comunicativas), siendo el grupo de enfermeras quienes obtuvieron puntajes más elevados. El resto de las dimensiones no presenta diferencias significativas $(\mathrm{H}=11.84, \mathrm{p}<.05)$. Cabe aclarar que para esta prueba se excluyó el grupo de psiquiatras ya que se trataba de un grupo demasiado pequeño como para realizar la comparación. En la tabla 4 se resumen los datos.

Por otro lado, en cuanto a los Años de experiencia, el grupo de profesionales con menos de 10 años de experiencia tiende a tener mayor Toma de Perspectiva $(\mathrm{U}=1641.50, \mathrm{p}<.05)$. En cuanto al resto de las dimensiones del constructo Empatía, no se encontraron diferencias significativas con la variable Años de Experiencia. Por otro lado, aquellas participantes con más de 10 años de experiencia en la profesión presentan mayores niveles de Habilidad Social $(\mathrm{U}=1559.00, \mathrm{p}<.05)$, componente que integra al constructo Habilidades Comunicativas; sin encontrar diferencias en el resto de las dimensiones ( $p>05$ ). En la Tabla 5 se resumen los resultados. 
Tabla 4

Diferencia entre Empatía y Habilidades Comunicativas y la Variable Categoría Profesional

\begin{tabular}{|c|c|c|c|c|c|c|}
\hline Variable & $\begin{array}{c}\text { R.P. } \\
\text { Psicóloga }\end{array}$ & $\begin{array}{c}\text { R.P. } \\
\text { Pediatra }\end{array}$ & $\begin{array}{c}\text { R.P. } \\
\text { Enfermera }\end{array}$ & $\begin{array}{l}\text { R.P. } \\
\text { Otra }\end{array}$ & $\boldsymbol{H}$ & $p$ \\
\hline Toma de Perspectiva & 76.09 & 67.40 & 54.85 & 72.92 & 2.529 & .470 \\
\hline Fantasía & 74.00 & 76.13 & 61.60 & 71.75 & .904 & .824 \\
\hline Preocupación Empática & 67.38 & 83.97 & 57.65 & 78.04 & 4.430 & .219 \\
\hline Malestar Personal & 61.90 & 88.57 & 71.30 & 80.67 & 8.581 & .035 \\
\hline Empatía total & 67.73 & 84.53 & 59.80 & 77.07 & 3.679 & .298 \\
\hline Comunicación Informativa & 68.36 & 89.47 & 76.15 & 72.03 & 3.238 & .356 \\
\hline Empatía & 80.14 & 53.97 & 65.20 & 69.99 & 5.864 & .118 \\
\hline Respeto & 82.06 & 59.43 & 67.55 & 65.84 & 6.916 & .075 \\
\hline Habilidad Social & 59.23 & 84.87 & 85.05 & 82.28 & 11.849 & .008 \\
\hline Hab. Com. Total & 69.74 & 74.40 & 77.15 & 74.35 & .544 & .909 \\
\hline
\end{tabular}

Nota: Hab. Com. Total: Habilidad comunicativa total. R.P.: Rango promedio.

Tabla 5

Diferencia entre Empatía y Habilidades Comunicativas y la Variable Años de Experiencia

\begin{tabular}{lcccc}
\hline Variable & $\begin{array}{c}\text { R.P. } \\
\text { Menos de 10 años }\end{array}$ & $\begin{array}{c}\text { R.P. } \\
\text { Más de 10 años }\end{array}$ & $\boldsymbol{D}$ \\
\hline Toma de Perspectiva & 78.37 & 61.04 & 1641.50 & .025 \\
Fantasía & 77.44 & 63.4 & 1738.50 & .070 \\
Preocupación empática & 72.17 & 76.9 & 2013.00 & .539 \\
Malestar Personal & 74.45 & 71.06 & 2052.50 & .661 \\
Empatía total & 77.2 & 64.04 & 1764.50 & .091 \\
Com. Informativa & 72.87 & 75.12 & 2086.00 & .771 \\
Empatía & 72.98 & 74.83 & 2098.00 & .808 \\
Respeto & 72.72 & 75.49 & 2071.00 & .712 \\
Habilidad Social & 67.85 & 87.98 & 1559.00 & .009 \\
Hab. Com. Total & 70.21 & 81.91 & 1807.50 & .133 \\
\hline
\end{tabular}

Nota. Com.: Comunicación. Hab. Com. Total: Habilidad comunicativa total.

Con respecto a atender a niños/as o a niños/as y cuidadores, se encontraron únicamente diferencias en la dimensión Empatía de Habilidades Comunicativas $(\mathrm{U}=1342, \mathrm{p}<.05)$, siendo el grupo de profesionales que atiende tanto a niños como a sus cuidadores quienes puntuaron más alto. Por último, no se encontraron diferencias en función del tipo de terapia $(\mathrm{p}>.05)$. 


\section{CONCLUSIÓN}

El objetivo de la investigación fue describir y asociar las variables empatía y habilidades comunicativas entre sí y con variables sociodemográficas en aquellas profesionales de la salud que trabajan con enfermedades crónicas pediátricas. Los resultados obtenidos permiten confirmar parcialmente la hipótesis planteada, ya que, si bien no existe una correlación significativa entre los constructos totales de empatía y habilidades comunicativas, sí es posible observar correlaciones directas entre algunas de sus dimensiones. Estos resultados coinciden de manera parcial con los que se obtuvieron en una investigación realizada por Monteza Olivera (2018) en donde se halló una asociación positiva entre la empatía y las habilidades comunicativas. Asimismo, ambas investigaciones registran un nivel medio de empatía en los encuestados, aunque resulta imposible generalizar esta coincidencia al constructo de habilidades comunicativas ya que, mientras en la presente investigación se observó un nivel alto en los participantes, la autora mencionada señala que en su muestra el nivel fue prioritariamente regular.

En cuanto a las variables sociodemográficas, la edad se asoció de forma negativa con la dimensión fantasía de empatía. En cambio, existe una relación significativa y directa con la dimensión preocupación empática, perteneciente al mismo constructo. Estos resultados coinciden con los hallados por Mejía de Díaz (2012) al concluir que existe una correlación positiva entre la edad y la compasión. Por su parte, se ha encontrado que, a mayor edad, mayores son los niveles en habilidades sociales (componente de las habilidades comunicativas). Estos datos discrepan con los resultados de Leal Costa et al. (2015), quienes han encontrado que todas las dimensiones de la EHC-PS correlacionan de manera negativa con la edad. Probablemente pueda atribuirse esta discrepancia a las muestras de estudio, ya que la presente investigación se centró exclusivamente en profesionales que atienden ECP, criterio no compartido con el estudio citado.

A propósito de la variable sociodemográfica Categoría Profesional, no se ha encontrado una correlación significativa con el constructo empatía total, es decir, no existen diferencias en la empatía según la profesión. No obstante, sí se ha observado que el grupo de pediatras presenta mayores niveles en una de sus dimensiones llamada malestar personal, indicando que experimentan mayor angustia y ansiedad ante las experiencias negativas del otro. Estos datos no concuerdan con los alcanzados por Putrino et al., (2018) quienes han observado que médicos y psicólogos profesionales se ven menos afectados emocionalmente por el malestar ajeno que el grupo de estudiantes de psicología que formaron parte de la muestra. Lejos de ser interpretado como una desventaja, este resultado da cuenta del avance de los profesionales en la adquisición de habilidades de regulación emocional que les permite hacer frente de manera adecuada a las demandas emocionales que surgen ante el encuentro con el dolor ajeno. Esta diferencia puede deberse al género de los participantes incluidos, ya que, en el estudio mencionado, se trabajó sobre 
una muestra mixta mientras que la presente investigación lo hizo sobre una población exclusiva de mujeres. Esto se debe a que trabajos sobre empatía han manifestado que las mujeres suelen puntuar más alto que los hombres (Quince et al., 2011).

Continuando con la variable Categoría Profesional, es posible afirmar que el constructo total de habilidades comunicativas tampoco difiere según la formación profesional, a excepción de la dimensión habilidad social que indica que las enfermeras han de tener mayor destreza asertiva. Estos datos se ajustan a los hallados por Leal Costa et al. (2015) quienes han llegado a la conclusión de que los médicos y enfermeros puntúan más alto en habilidad social que los auxiliares de enfermería. Según los resultados de dicha investigación, también es posible afirmar que las dimensiones de las habilidades comunicativas se relacionan con una sensación de mayor realización laboral, evitando así efectos adversos como el síndrome de burnout. Por ende, si bien el tipo de profesión no influye en los niveles de habilidades comunicativas, éstas sí repercuten en un estado de mayor bienestar independientemente de la profesión.

En lo que respecta a la variable sociodemográfica Años de Experiencia, los resultados indican que no correlaciona de manera significativa con la empatía total de las profesionales. Empero, sí se observa que quienes cuentan con menos de 10 años de trayectoria presentan mayor habilidad para comprender el punto de vista de otra persona (dimensión toma de perspectiva). Estos resultados coinciden parcialmente con los de Putrino et al. (2018) quienes hallaron que la toma de perspectiva es mayor en quienes no alcanzan la década de experiencia. Sin embargo, la diferencia con quienes tienen más de 11 años de ejercicio en la profesión es sumamente pequeña. Esto condice con investigaciones como la de Quince et al. (2011) que sugieren que el componente cognitivo de la empatía se mantiene estable a lo largo de los años, mientras que las modificaciones son observables principalmente en el componente afectivo, el cual tiende a disminuir. De todas formas, los resultados aquí obtenidos no permiten adherir ni confirmar esta última conclusión.

En sintonía con estas ideas, la variable Años de Experiencia tampoco influye en los niveles de habilidades comunicativas total, aunque aquellas profesionales con más de 10 años de experiencia puntuaron más alto en la dimensión habilidades sociales. Tiuraniemi et al. (2011) se interesó por evaluar las competencias comunicativas e interpersonales en estudiantes de medicina y psicología y ha encontrado que tras un entrenamiento basado en role playing, clases teóricas y demás ejercicios, ambos grupos han advertido un aumento en sus destrezas sociales. $\mathrm{Si}$ formaciones breves afectan positivamente el desarrollo de estas habilidades, entonces más de 10 años de ejercicio de la profesión son suficientes para justificar el resultado obtenido.

Considerando que la muestra estuvo compuesta por participantes de diversas profesiones, se ha evaluado si en el caso de las psicólogas la variable 
sociodemográfica Tipo de Terapia generaba cierta influencia sobre las variables estudiadas. Según lo observado, esto no es así. El posicionamiento teórico de cada terapeuta ante el abordaje a pacientes con enfermedades crónicas pediátricas no influye en sus niveles de empatía y habilidades comunicativas total. Estos resultados se distinguen de los logrados por Casari et al. (2017) quienes, en una investigación realizada con terapeutas que atendían a niños con TEA, han encontrado que aquellos que adhieren a un enfoque integrativo presentan un mayor compromiso y acercamiento emocional con los pacientes. Además, se encontró que los psicólogos cognitivo conductuales realizan tratamientos más pautados y menos espontáneos, además de sostener un encuadre más distante emocional y comunicativamente. Es importante resaltar que existen diferencias en las investigaciones, como la variable estudiada y la población seleccionada, que podrían traducirse en una discrepancia entre resultados.

Por último, en lo que respecta a la variable sociodemográfica Atención a Niños o a Niños y Cuidadores, más de la mitad de la muestra ha manifestado trabajar con ambas partes, resultado que se diferencia de los alcanzados por De la Iglesia et al. (2012) al afirmar que el $90 \%$ de las interacciones se da entre el personal sanitario y padres de niños asmáticos, incluyendo al niño sólo en el 10\% restante de las situaciones. Las dimensiones y el constructo total de empatía no varían según si la atención es exclusiva a niños o a éstos y sus cuidadores. En este caso, sí se observa una puntuación significativa en la dimensión empatía del constructo habilidades comunicativas en quienes contemplan tanto a los pequeños como a los adultos en el proceso de atención. Estos datos nuevamente se contradicen con el estudio ya mencionado, en el cual se ha encontrado que un $62 \%$ de las consultas suelen ser interrumpidas y que gran parte de la información se recoge en el proceso de admisión previo a la consulta médica, restando tiempo y dedicación exclusiva al paciente y su malestar. Estas discrepancias pueden atribuirse a las diferencias en el enfoque de la investigación.

Como toda investigación, el presente estudio se ha visto condicionado por ciertas limitaciones. Entre ellas, se puede mencionar la desproporción en la cantidad de participantes pertenecientes a cada categoría profesional, como así también, en la cantidad de personas que han reportado trabajar con cada enfermedad crónica. A su vez, la selección de la muestra fue relativamente pequeña y de corte transversal, lo que dificulta alcanzar una generalidad más abarcativa en los resultados. Sin embargo, amerita resaltar como fortaleza, que la investigación ha logrado evaluar una variable como habilidades comunicativas en una muestra novedosa, siendo que, hasta el momento, la mayoría de las investigaciones han sido realizadas únicamente sobre muestras de enfermeros o médicos.

Se recomienda para futuras investigaciones lograr una homogeneización en los grupos estudiados como así también limitar las enfermedades crónicas seleccionadas, para que los resultados sean más específicos y arrojen información útil 
para la práctica diaria. También se aconseja que en próximas oportunidades se realice un estudio longitudinal para comparar a una misma muestra en el tiempo y así observar los cambios que se producen en las variables de estudio. En último lugar, se recomienda la incorporación de otras variables de estudio no sólo en los profesionales de la salud sino también sobre los propios pacientes con enfermedades crónicas, para seguir haciendo ciencia en un área tan incipiente como necesaria.

A partir de la información presentada, se sugiere la incorporación de talleres o espacios de formación que permitan a los profesionales potenciar habilidades innatas o adquirirlas a partir de su aprendizaje, equiparando así la adquisición de competencias sociales con la de competencias técnicas. Cuando la comunicación entre los profesionales de la salud y los pacientes (pediátricos o no) hace foco en las necesidades de este último, tiene entonces las propiedades necesarias para convertirse en un potencial recurso terapéutico.

\section{Conflictos de interés}

Los autores declaran que no existen conflictos de interés en la elaboración del manuscrito.

\section{Financiamiento}

Autofinanciado.

\section{REFERENCIAS}

Universidad Favaloro (2016). Trastornos del Espectro Autista: afectan a 1 de cada 68 chicos. https://doi.org/10.26820/recimundo/2.(3).julio.2018.650-665

Asociación Argentina de Pediatría. (2018). 2 de Abril: Día mundial de concientización sobre el autismo. https://doi.org/10.18356/1730c5b8-es

Asociación Médica Mundial (2000). Declaración de Helsinki. https://medicina.udd.cl/ centro-bioetica/files/2010/10/declaracion_helsinski.pdf

Bermejo, C. (2012). Empatía terapéutica: la compasión del sanador herido. Desclée de Brouwer.

Borrell Carrió, F. (2011). Empatía, un valor troncal en la práctica clínica. Medicina Clínica, 136(9), 390-397. https://doi.org/10.1016/j.medcli.2009.06.032

Casari, L., Assennato, F. y Grzona, S. (2017). Estilo Personal del Terapeuta de psicólogos que trabajan en Autismo. Revista de Psicoterapia, 28(108), 169-188. https://doi. org/10.33898/rdp.v28i108.164

Davis, M. H. (1980). A multidimensional approach to individual differences in empathy. JSAS Catalog of Selected Documents in Psychology, 10(4), 85-104. 
De la Iglesia, B., Roselló, M.R. y Verger, S. (2012). La relación comunicativa entre el profesional sanitario y el paciente asmático en Pediatría. Revista de Investigación en Educación, 10(1), 45-59. https://doi.org/10.3145/epi.2012.jul.10

Echeburúa, E., Salaberría, K., de Corral, P. y Polo López, R. (2010). Terapias Psicológicas Basadas en la Evidencia: limitaciones y retos del futuro. Revista Argentina de Clínica Psicológica, 19(3), 247-256. https://doi.org/10.5944/rppc.vol.15.num.2.2010.4088

Etchezahar, E., Prado-Gascó, V., Jaume, L., \& Brussino, S. (2014). Validación argentina de la escala de Orientación a la Dominancia Social (SDO). Revista Latinoamericana de Psicología, 46(1), 35-43.

Georgi, E., Petermann, F. y Schipper, M. (2015). Do empathic abilities change throughout the course of becoming a psychotherapist? Zeitschriftfür Neuropsychologie, 26(3), 1-9.

Gil-Monte, P. R. (2005). Factorial validity of the Maslach Burnout Inventory (MBI-HSS) among Spanish Professionals. Revista de Saúde Pública, 39, 1-8

Gismero, E. (2010). EHS Escala de Habilidades Sociales Manual. TEA.

Hospital de Pediatría Garrahan (2010). Problemáticas complejas en la infancia: el impacto de enfermedades crónicas en la familia y comunidad [Archivo PDF]. https://www.sap. org.ar/docs/congresos/2010/ambulatoria/rodriguez_rodriguez_problematicas.pdf

Lambert, M. J. (2013). Bergin and Garfield's Handbook of Psychotherapy and Behavior Change. Wiley.

Leal-Costa, C., Díaz-Agea, J.L., Tirado-González, S., Rodríguez-Marín, J. y van-der Hofstadt, C.J. (2015). Las habilidades de comunicación como factor preventivo del síndrome de Burnout en los profesionales de la salud. Anales del Sistema Sanitario de Navarra, 38(2), 213-223. https://doi.org/10.4321/s1137-66272015000200005

Leal-Costa, C., Tirado-González, S., Rodríguez-Marín, J. y van-der Hofstadt, C. J. (2016). Psychometric properties of the Health Professionals Communication Skills Scale (HPCSS). International Journal of Clinical and Health Psychology, 16(1), 76-86. https://doi.org/10.1016/j.ijchp.2015.04.001

Lejarraga, H. (2006). La atención pediátrica de pacientes crónicos, una práctica necesaria. Archivos Argentinos de Pediatría, 104(1), 62-63.

Liga Argentina contra la Epilepsia. (2020). La epilepsia y los niños. https://doi.org/10.2307/j. ctv893gxz.44

Mejía de Díaz, M.A. (2012). Conducta empática en los estudiantes de ciencias de la salud, Universidad de los Andes, Mérida, Venezuela [Tesis de Licenciatura, Universidad Autónoma de Madrid]. https://doi.org/10.22209/rt.ve2019a10

Mercer S. W. y Reynolds, W. J. (2002). Empathy and quality of care. British Journal of General Practice, 52, 9-13.

Monteza Olivera, D.R. (2018). Relación entre las Habilidades Comunicativas y la Empatía de los internos de medicina del Hospital Regional Honorio Delgado [Tesis de licenciatura, Universidad Católica de Santa María]. https://doi.org/10.26696/sci. epg.0023 
Müller, M., Ungaretti, J. y Etchezahar, E. (2015). Evaluación multidimensional de la empatía: Adaptación del Interpersonal Reactivity Index (IRI) al contexto argentino. Revista de Investigación en Psicología Social, 3(1), 42-53.

Organización Mundial de la Salud. (1 de junio de 2018). Enfermedades no Transmisibles. https://www.who.int/es/news-room/fact-sheets/detail/noncommunicable-diseases

Putrino, N., Tabullu, A., Mesurado, B. y Richaud di Minzi, M. C. (2018). Are there differences in health professionals' empathy due to academic and clinical experience? Current Psychology, 40, 647-654. https://doi.org/10.1007/s12144-018-9980-x.

Quesada Conde, A. B, Justicia Díaz, M. D., Romero López, M. y García Berbén, T. (2014). La enfermedad crónica infantil: repercusiones emocionales en el paciente y la familia. Revista de Psicología, 4(1), 569-576. https://doi.org/10.17060/ijodaep.2014. n1.v4.832

Quince, T., Parker, A., De Madera, D. y Benson J. (2011). Stability of empathy among undergraduate medical students: A longitudinal study at one UK medical school. BMC Medical Education, 11(90), 1-9. https://doi.org/10.1186/1472-6920-11-90

Registro Oncopediátrico Hospitalario Argentino. (2018). Registro Oncopediátrico argentino. Tendencia de incidencia 2000-2016. Supervivencia a 5 años 2005-2011. Tendencia de supervivencia secular: 200o-04,2005-09 y 2010-14. https://www.argentina.gob.ar/salud/instituto-nacional-del-cancer/institucional/roha. https:/doi. org/10.35376/10324/30287

Rogers, C. (1951). Terapia centrada en el cliente. Paidós.

Tiuraniemi, J., Läärä, R., Kyrö, T. y Lindeman, S. (2011). Medical and psychology students' self-assessed communication skills: A pilot study. Patient Education and Counseling, 83(2), 152-157.

Universidad Favaloro (2017). Confirman que el déficit de atención es un "trastorno cerebral" y piden no etiquetar a los chicos. http://www.favaloro.edu.ar/8372-2/

Wyatt, K. D., List, B., Brinkman, W. B., Prutsky Lopez, G., Asi, N., Erwin, P., Wang, Z., Domecq Garces, J. P., Montori, V.M. y LeBlanc, A. (2015). Shared Decision Making in Pediatrics: A Systematic Review and Meta-analysis. Academic Pediatrics, 15(6), 573-583.

Yalom, I. D. (2002). El don de la terapia: carta abierta a una nueva generación de terapeutas y a sus pacientes. Emecé.

Zwaanswijk, M., Tates, K., Dulmen, S., Hoogerbrugge, P. M., Kamps, W. A., Beishuizen, A. y Bensing, J. M. (2011). Communicating with children patients in pediatric oncology consultations: a vignette study on child patients, parents anda survivors communication preferences. Psycho Oncology, 20(3), 269-277. https://doi.org/10.1002/ pon.1721 
